\title{
Progress in Endoscopic Treatment of Upper Gastrointestinal Bleeding
}

\author{
Qinghai $\mathrm{Fu}^{1^{*}}$, Qingming $\mathrm{Wu}^{2}$, Jiguang $\mathrm{Kou}^{1}$, Jinjun $\mathrm{Li}^{3}$ \\ ${ }^{1}$ Department of Gastroenterology, Xiaogan Central Hospital Affiliated to Wuhan University of Science and Technology, Xiaogan, \\ China \\ ${ }^{2}$ Department of Gastroenterology, Tianyou Hospital Affiliated to Wuhan University of Science and Technology, Wuhan, China \\ ${ }^{3}$ Department of Surgery, College of Medicine, Wuhan University of Science and Technology, Wuhan, China \\ Email: *1198868221@qq.com
}

How to cite this paper: Fu, Q.H., Wu, Q.M., Kou, J.G. and Li, J.J. (2018) Progress in Endoscopic Treatment of Upper Gastrointestinal Bleeding. International Journal of Clinical Medicine, 9, 234-242. https://doi.org/10.4236/ijcm.2018.94020

Received: March 17, 2018

Accepted: April 16, 2018

Published: April 19, 2018

Copyright $\odot 2018$ by authors and Scientific Research Publishing Inc. This work is licensed under the Creative Commons Attribution International License (CC BY 4.0).

http://creativecommons.org/licenses/by/4.0/

\begin{abstract}
Due to the increase in the incidence of upper gastrointestinal bleeding, the treatment methods are more and more diversified. With the popularization of endoscopic techniques and the wide range of endoscopic hemostasis methods, endoscopy has become an important method for the treatment of upper gastrointestinal bleeding, and greatly updated the treatment concept of upper gastrointestinal bleeding. In this paper, we reviewed the literature reports of endoscopic treatment of upper gastrointestinal bleeding in recent years.
\end{abstract}

\section{Keywords}

Upper Gastrointestinal Bleeding, Endoscopy, Treatment

\section{Introduction}

Upper gastrointestinal bleeding is a common acute and severe disease in the digestive department. It mainly includes the digestive tract above the ligament of Treitz, including bleeding caused by esophageal, gastric, duodenalor pancreatic and gallbladder lesions. Massive bleeding refers to blood loss exceeding $1000 \mathrm{ml}$ or $20 \%$ of circulating blood volume within a few hours [1]. Its clinical manifestations are mainly vomiting blood and/or black feces, and shock caused by acute peripheral circulation failure. The mortality rate is extremely high, even up to $12 \%-35 \%$ of elderly patients [2] [3]. Clinically, the causes are often divided into two categories: non-variceal bleeding and variceal bleeding. Endoscopic treatment mainly refers to the use of endoscopic hemostatic drug injection or other methods to get hemostasis. Now this article reviews recent domestic and foreign literature to summarize the progress of endoscopic treatment of upper gastroin- 
testinal bleeding.

\section{Endoscopic Treatment of Non-Variceal Bleeding}

1) Local drug spraying: This method is simple, safe and effective, and is often used in bleeding lesions. Commonly used drugs include: a) Norepinephrine: Select $8 \%$ concentration of norepinephrine in an ice-cold saline solution to spray on the bleeding lesions to stimulate blood vessel contraction by stimulating the capillary vasospasm receptor to make hemostasis; b) Thrombin: Thrombin is an important effector protease in the blood coagulation cascade. Local spraying under the endoscope can directly act on plasma fibrinogen, accelerate the formation of fibrin, and cover the surface of the lesion to achieve hemostasis; $c$ ) Monsell's solution: It contains Alkaline ferric sulfate, which has a strong convergence Coagulate blood, resulting in the formation of a protective film attached to the wound and achieve hemostasis [4]. d) Biological protein glue: It is a novel hemostatic drug consisting of fibrinogen, thrombin, fibrinolytic inhibitor and calcium chloride extracted, which is extracted from animal blood and get virus inactivation treatment. Using endoscopy drugs can be sprayed on the lesions to form a thin layer of milky white glue, which can bind the wounds and stop venous bleeding. The protein gel also has good tissue compatibility, less adverse reactions and can promote tissue growth and repair [5]. e) Hemostatic powder: More and more new types of hemostatic drugs are widely used in clinical practice. Commonly used are hemostatic powders extracted from plant ingredients, such as PerClotTM, AristATMAH and composite microporous polysaccharide powder from abroad. Polysaccharide hemostatic powder has very good dehydration ability, which can dehydrate and concentrate the blood quickly, so that the blood components such as platelets and fibrin can accumulate rapidly, and thus form a kind of "gel-like" blood clot, which can be adhere to lesions to stop bleed. In addition, the hemostatic powder does not contain animal protein components, it can be degraded quickly in the body, and had a low incidence of adverse reactions, and it also has good compatibility with the human body. Zhao [6] reported that 35 patients with non-variceal upper gastrointestinal bleeding were treated with this method. The success rate of this method was $100 \%$, and the average time required to stop bleed was $(34 \pm 2)$ minutes, Its therapeutic effect is better than traditional medicine. No rebleeding, allergic reactions or other adverse reactions were appeared after surgery. TC-325 (Hemospray) is a mineral-based hemostatic powder. Its hemostatic mechanism is also the rapid absorption of the fluid component of blood, it can promote blood concentration, solidification, forming a gel-like mechanical barrier, activating clotting reactions. In a prospective study conducted by Haddara [7], it collected 75 patients of bleeding with ulcer, tumor, and endoscopic hemorrhage. The effective rate of hemostasis with TC-325 was 96.5\%, the effective rate of first line treatment was $96.8 \%$, and the effective rate of remedial treatment was $96.3 \%$.

2) Local drug injection: Endoscopic drug injection hemostasis is commonly 
used in the treatment of active bleeding. a) Adrenaline: It is the most commonly used drug, which can contract the blood vessels around the lesions to achieve the purpose of hemostasis. It can make the tissue around the lesion swollen and exert the role of mechanical compression hemostasis. b) Sclerosing Agent: $1 \%$ aethoxysklerol (AS) and 5\% sodium morrhuate (SM) are most commonly used sclerosing agent, the injection site is often selected around the submucosa of the lesion, the mechanism is that the sclerosing agent can cause local tissue swelling, initiate local tissue swelling, increase the blood pressure of the lesion, compress the peripheral blood vessels, accelerate the formation of blood vessel thrombosis, and promote the fibrosis of the blood vessels and surrounding tissues to achieve hemostasis. However, in the treatment of sclerotherapy, attention should be paid to the control of the dose, speed, and depth of injection in order to prevent the occurrence of erosions and ulcers, and even serious complications such as perforation at the injection site [8]. c) Anhydrous alcohol: It is cheap and easy to obtain. It is injected around the bleeding lesions through endoscope. Because of its dehydration, the tissue surrounding the lesion is degenerated and necrosed to achieve hemostasis.

3) Heat coagulation: It is often applied to lesions with erosions, ulcers or vascular stumps. Clinically applied Heat coagulation methods are often classified as contact and noncontact. a) Contact heat coagulation hemostasis: it contains heat probe (HP), hemostatic forceps, high-frequency electrocoagulation (single pole, bipolar and multipole electrocoagulation). The working mechanism of HP is to release the thermal energy through contact with the tissue, which makes the lesion tissue and blood vessels degenerate and necrosis and serves the purpose of hemostasis. Domestic studies [9] have shown that the temperature can achieve effective hemostasis at $150^{\circ} \mathrm{C}-180^{\circ} \mathrm{C}$, and when the temperature continues to rise to $200^{\circ} \mathrm{C}$ around $15 \mathrm{~s}$, there is no perforation occurred. High-frequency electrocoagulation is the use of high-frequency electric to treat hemorrhagic lesions, which can increase its local temperature quickly, promote protein degeneration, surrounding tissue edema and accelerate vasoconstriction, The blood vessels become thinner and occluded, accelerating thrombus formation, thereby achieving hemostasis. Some scholars [10] have reported that for patients with non-variceal gastrointestinal bleeding, the immediate rate of hemostasis can be as high as $100 \%$, and the rate of rebleeding is only $3.7 \%$. b) Non-contact heat coagulation hemostasis: Argon Plasma Coagulator (APC), Laser Photocoagulation and so on. APC is often applied to peptic ulcers, tumors and vascular stump hemorrhages [11]. It transmits high-frequency energy to the lesion tissue by emitting argon gas. The surface of the tissue can coagulate to achieve hemostasis. When the focus tissue coagulates and dries, it loses its ability to conduct electricity, which limits the energy to damage the normal tissue [12]. And because APC is a non-contact electrocoagulation technology, it can avoid adhesion of the electrode to the surface of the lesion and prevent the coagulated tissue from rebleeding due to avulsion. Laser treatment commonly used carbon dioxide laser, 
holmium laser and so on. The energy generated by the $\mathrm{CO}_{2}$ laser is easily absorbed by water, so the penetration depth is limited, but the $\mathrm{CO}_{2}$ laser cannot be conducted through the optical fiber, which limit the application of hemostasis through the endoscope; Holmium laser is a pulsed solid-state laser with yttrium aluminum garnet (YAG) as the activation medium. Its wavelength is $2.1 \mathrm{um}$, has a high absorption coefficient for water, because human soft tissue contains abundant water, it can effectively burn and coagulate the diseased tissue. In addition, the short wavelength of the YAG laser can be transmitted through the soft fiber and the blind zone is small during the operation, so it is particularly suitable for the operation of the endoscope under the digestive tract [13].

4) Frozen hemostasis: The most commonly used are liquid nitrogen and carbon dioxide, often need freezing rod to contact and spray the frozen gas to cool the lesion tissue rapidly and coagulation, necrosis, to achieve the purpose of hemostasis, but the this method requires special equipment, complex operation, there are fewer clinical applications currently.

5) Mechanical hemostasis: a) Metal Hemoclip: Especially suitable for the situation where the blood vessel is exposed in the bleeding lesion. Under the guidance of the endoscope, the clip head is adjusted to be perpendicular to the bleeding site. After the disassembling of the clip, the blood vessel is clamped by the helper's operation. After the wound is healed, the metal clip will fall off on its own after a few weeks and will be excreted by the feces. Studies have shown that for active arteriole hemorrhage, a very significant hemostatic effect can be achieved through the closure of mucosal vascular stumps [14]. For the digestive tract tumor and the esophagus ulcer after radiotherapy, the metal clip is easy to fall off because of the brittleness of the tissues, so it is not suitable for the hemostasis using this method. b) Ligation Method: commonly used a leather ring and a nylon rope, this method is often applied to include Dieulafoy's disease, Mallory Weiss syndrome, comprehensive hemorrhage after polypectomy and mucosal vascular malformation and other causes of endoscopic treatment [15]. The mechanism is mainly through negative pressure to attract lesions and surrounding tissues, and use elasticity to block the blood supply of lesions, resulting in ischemic necrosis of ligature tissue. After a few days, the tissue was shedding together with the ring. c) Membrane-covered Metallic Stent: For postoperative bleeding such as endoscopic sphincterotomy (EST). It is often combined with massive bleeding, deeper lesions, poor operation vision, apparatus difficult to reach, etc. Some scholars [16] have reported about 4 cases of severe bleeding after EST using Membrane-covered Metallic Stent can be effective in the hemostasis, and has a simple operation, minimally invasive, rapid recovery, which provides new idea for the treatment of upper gastrointestinal bleeding. d) Endoscopic suture technique: Overstitch is a new type of endoscopic suturing system. Chiu [17] and other scholars use endoscopic suturing technology to treat patients with large hemorrhagic gastric ulcers that cannot tolerate surgical treatment. This equipment consists of a suturing system. This equipment is composed of a 
suture system and fixed exchange system. The ulcer is first positioned through the endoscope, then the suturing device is used in conjunction with the needle and the needle holder to start suturing from the bottom of the lesion. Furthermore, the lesions are kept away from the acidic environment to achieve hemostasis, but this device is expensive and has not been widely used clinically, so the effectiveness and practicality of the devices need to be further explored.

\section{Endoscopic Treatment of Variceal Bleeding}

1) Endoscopic injection sclerotherapy (EIS): Commonly used sclerosants are $5 \%$ sodium morrhuate (SM), $1.0 \%$ to $1.5 \%$ sodium tetradecylsulfonate (STS) and $1 \%$ aethoxysklerol (AS), anhydrous alcohol, etc. The injection method includes varicose vein para-infusion, varicose vein injection and co-injection. The mechanism is to make the varicose venous produce aseptic chemical inflammation through endoscopic injection of sclerosingagent, adhesion and thrombosis of vascular mucosa, resulting in blockage of lumen. In addition, the surrounding mucous membrane of the vein was necrotic and fibrotic, and the scar was formed to oppress the varicose veins, blocking the blood flow to reduce the venous pressure and to achieve the aim of hemostasis. The recurrence of esophageal varices is related to the size of the paraesophageal vein and the number of perforating branches. When the sclerosant enters the perforating branch with the bloodstream, it will also cause occlusion, which will reduce the subsequent recurrence rate [18]. However, EIS treatment is also related with complications, such as ulcer of the treated area, esophageal stricture, perforation, and even the possibility of ectopic embolization.

2) Endoscopic Variceal Ligation (EVL): The mechanism is to attach the rubber band to the root of the varicose vein through the suction under the endoscope, which can block the blood flow and form a thrombus to gradually occlude the blood vessel. The varicose veins and their surrounding mucosal tissues lost their blood supply, and they developed ischemic necrosis and abscission. The lesions formed superficial ulcers, scars after healing, which can stop and preventing bleeding. At present, there are many applications such as single ligation, multiple continuous ligation and nylon ligation, but the most widely used is multi-striping ligation. For severe varicose veins, one-time completion of intensive ligation at more than 7 target points is more effective than standard ligation, acute hemostasis is faster and the effect is better [19], However, EVL only has a good sealing effect on the superficial varicose veins, it can not effectively block the submucosal traffic branches, resulting in a higher recurrence rate of varicose veins.

3) Tissue Adhesive Embolization: The main component of commonly used tissue adhesives is $\mathrm{N}$-cyanoacrylate (Hitoacryl). Due to the effect of a small amount of anions in the blood and tissue fluids, it can instantly polymerize and solidify after contacting with blood, so as to block blood vessels and prevent bleeding of varicose veins. Therefore, tissue injection for varicose veins is widely 
applied in clinic. Studies [20] have shown that the early hemostasis rate using this method is as high as $98.4 \%$. But the tissue adhesive also has its disadvantages, it can be cured in contact with air, plug the needle easily or even damage the pipeline and endoscopic tissue glue. For the blood vessels that are not fully embolized, it is easy to form ulcer and rebleeding [21]. Secondly, tissue adhesive can not induce local inflammatory reaction, vascular fibrosis, and prevent the recurrence of varicose veins. Therefore dilution method and "sclerosant-tissue adhesive-sclerosant" called sandwich method are often used to improve its deficiency. Dilution method often uses iodine, inhaling a little fat-soluble iodine before the injection to form a thin film in the wall of the needle, after the injection of adhesive, reinject a little saline can effectively prevent the tissue adhesive damaging the needle. The "sandwich" method is more advantageous, sclerosant can be absorbed by the body, will not make the body produce a degumming reaction. This method can make varicose veins reduce or occlusion effectively, significantly reduce the recurrence rate of patients with varicose veins and rebleeding rate. A meta-analysis [22] showed that the combination of sclerosant and tissue adhesive was more effective than single tissue adhesive used in the treatment of gastric varicose veins.

4) Endoscopic Ultrasonography (EUS): EUS is performed through a highfrequency ultrasound probe placed at the end of endoscope. It can not only observe the internal shape of the lumen directly through endoscopy, but also perform histology of the digestive cavity' wall through ultrasound, It has more advantages than the simple endoscopic and ultrasonic diagnose [23]. EUS is not only able to observe esophageal and gastric fundus lesions, but also to understand esophageal perforating veins and para veins at the same time. Early assessment of the progress of the patients with chronic liver disease can guide them to prevent gastrointestinal bleeding early. A 6-year research [24] also showed that the placement of coils under the guidance of EUS and combined with injection method can effectively reduce the risk of heterotopic embolization while effectively treating esophageal and gastric varices. It plays an effective role in active bleeding and primary prevention of gastric varices.

5) Argon Plasma Coagulator (APC): As mentioned above, APC transfers high-frequency energy to the target tissue, which causes the target tissue to coagulate and degenerate, and accelerates the fibrosis of the distal esophageal mucosal layer to inhibit the progress of capillary revascularization and the branch veins. Kamal [25] reported a group study of 40 patients with varicose veins. The recurrence rate of varicose veins in patients undergoing EVL with APC treatment was significantly lower than that of patients treated with EVL alone $(\mathrm{P}<$ 0.001). Therefore, APC was used to prevent esophageal veins is a certain effect in the prevention of varicose veins.

\section{Summary and Prospect}

Due to the complex cause of upper gastrointestinal bleeding, it is very important 
to identify the etiology and formulate an effective treatment plan in time. With the continuous progress of digestive endoscopy, the application of endoscopy in diagnosis and treatment is constantly improving. Because of its convenient operation, less trauma, high safety and effectiveness, rapid postoperative recovery, endoscopy plays an increasingly important role in the treatment of upper gastrointestinal bleeding, and gradually become the first choice of diagnosis and treatment of upper gastrointestinal bleeding. Although the application of endoscopic technology is increasingly diversified, it is still necessary to continue to develop newer, safer and more effective endoscopic techniques in the future to improve the survival rate of patients.

\section{References}

[1] Liu, S.Q., Qu, X.W., Zhang, S.W., et al. (2012) The Experience of Laparoscopic Treatment for Acute Large Gastroduodenal Hemorrhage. Journal of Laparoscopic Surgery, 7, 489-491.

[2] Christensen, S., Riis, A., et al. (2007) Short-Term Mortality after Perforated or Bleeding Peptic Ulcer among Elderly Patients: A Population-Based Cohort Study. BMC Geriatrics, 7, 8. https://doi.org/10.1186/1471-2318-7-8

[3] Rockall, T.A., Logan, R.F., Devlin, H.B. and Northfield, T.C. (1995) Incidence of and Mortality from Acute Upper Gastrointestinal Haemorrhage in the United Kingdom. Steering Committee and Members of the National Audit of Acute Upper Gastrointestinal Haemorrhage. BMJ, 311, 222-226.

https://doi.org/10.1136/bmj.311.6999.222

[4] Zhang, J.J., Guo, J.H. and Wang, F.F. (2014) Endoscopic Monsell's Curative Effect Observation of Hemorrhage of Digestive Tract Tumor Mergers. Zhejiang Clinical Medicine Journal, 16, 1797-1798.

[5] Li, Z. (1998) The Principle of Action of Fibrin Glue and Its Clinical Application and Adverse Reaction. West China Journal of Pharmaceutical Sciences, 14, 34.

[6] Zhao, X.Y., Zhang, B.P., et al. (2017) Application of Composite Microporous Polysaccharide Powder in Therapy of Non-Varicose Veins of Gastrointestinal Bleeding. The Journal of Practical Medicine, 33, 3927-3929.

[7] Haddara, S., Jacques, J., et al. (2016) A Novel Hemostatic Powder for Upper Gastrointestinal Bleeding: A Multicenter Study (the "GRAPHE" Registry). Endoscopy, 48, 1084-1095.

[8] Cheng, C.L., Liu, N.J., Lee, C.S., et al. (2004) Endoscopic Management of Dieulafoy Lesions in Acute Nonvarieeal Upper Gastrointestinal Bleeding. Digestive Diseases and Sciences, 49, 1139-1144. https://doi.org/10.1023/B:DDAS.0000037801.53304.5c

[9] Yu, L. and Wu, S.H. (1995) Experimental Study of Direct Current Heatprobe Hemostasis for Gastric Mucosal Bleeding. Chinese Journal of Medical Physics, 12, 86-89.

[10] Kataoka, M., Kawai, T., Hayama, Y., et al. (2013) Comparison of Hemostasis Using Bipolar Hemostatic Forceps with Hemostasis by Endoscopic Hemoclipping for Nonvarieeal Upper Gastrointestinal Bleeding in a Prospective Nonrandomized Trial. Surgical Endoscopy, 27, 3035-3038. https://doi.org/10.1007/s00464-013-2860-4

[11] Canard, J.M. and Védrenne, B. (2001) Clinical Application of Argon Plasma Coagulation in Gastrointestinal Endoscopy: Has the Time Come to Replace the Laser? Endoscopy, 33, 353-357. https://doi.org/10.1055/s-2001-13686 
[12] Conway, J.D., Adler, D.G., Diehl, D.L., et al. (2009) Endoscopic Hemostatic Devices. Gastrointestinal Endoscopy, 69, 987-996. https://doi.org/10.1016/j.gie.2008.12.251

[13] Nishioka, N.S., Domankevitz, Y., Flotte, T.J., et al. (1989) Ablation of Rabbit Liver, Stomach, and Colon with a Pulsed Holmium Laser. Gastroenterology, 96, 831-837. https://doi.org/10.1016/0016-5085(89)90909-8

[14] Arima, S., Sakata, Y., Ogata, S., et al. (2010) Evaluation of Hemostasis with Soft Coagulation Using Endoscopic Hemostatic Forceps in Comparison with Metallichemoclips for Bleeding Gastric Ulcers: A Prospective Randomized Trial. Gastroenterology, 45, 501-505.

[15] Keohane, J., Berro, W., Harewood, G.C., et al. (2013) Band Ligation of Gastric Antral Vascular Ectasia Is a Safe and Effective Endoscopic Treatment. Digestive Endoscopy, 25, 392-396. https://doi.org/10.1111/j.1443-1661.2012.01410.x

[16] $\mathrm{Wu}$, J. and $\mathrm{Hu}, \mathrm{B}$. (2012) Covered Self-Expandable Metal Stent for Uncontrolled Bleeding after Endoscopic Sphincterotomy. Chinese Journal of Digestive Endoscopy, 4, 194-196.

[17] Chiu, P.W., Chan, F.K. and Lau, J.Y. (2015) Endoscopic Suturing for Ulcer Exclusion in Patients with Massively Bleeding Large Gastric Ulcer. Gastroenterology, 149, 29-30. https://doi.org/10.1053/j.gastro.2015.04.054

[18] De Paulo, G.A., AIdengh, J.C., Nakao, F.S., et al. (2006) Treatment of Esophageal Varices: A Randomized Controlled Trial Comparing Endoscopic Sclerotherapy and EUS-Guided Sclerotherapy of Esophageal Collateral Veins. Gastrointestinal Endoscopy, 63, 396-402. https://doi.org/10.1016/j.gie.2005.10.039

[19] Yang, K.-Z., Zhu, Z.-M., et al. (2017) Comparison of the Efficacy of Non-Dense Ligation and Dense Ligation of Esophageal Varices on Patients with Severe Esophageal Varices. Journal of Hunan Normal University (Medical Science), 14, 133-135.

[20] Kang, E.J., Jeong, S.W., Jang, J.Y., et al. (2011) Long-Term Result of Endoscopic Histoacryl (N-butyl-2-cyanoacrylate) Injection for Treatment of Gastric Varices. World Journal of Gastroenterology, 17, 1494-1500.

[21] Cheng, L., Wang, Z., et al. (2007) Treatment of Gastric Varices by Endoscopic Sclerotherapy using Butyl Cyanoscrylate: 10 Years' Experience of 635 Cases. Chinese Medical Journal, 120, 2081-2085.

[22] Zhou, Y., Li, P., et al. (2017) Efficacy and Safety of Tissue Adhesive Combined with Lauromacrogol for Gastric Varices: A Systematic Review. Medical Recapitulate, 23, 3302-3307.

[23] May, A. and Ell, C. (2011) Gastroenterological Endoscopy. Deutsche Medizinische Wochenschrift, 136, 206-208. https://doi.org/10.1055/s-0031-1272511

[24] Bhat, Y.M., Weilert, F., Fredrick, R.T., et al. (2016) EUS-Guided Treatment of Gastric Fundal Varices with Combined Injection of Coils and Cyanoacrylate Glue: A Large U.S. Experience over 6 Years (with Video). Gastrointestinal Endoscopy, 83, 1164-1172. https://doi.org/10.1016/j.gie.2015.09.040

[25] Kamal, A., Abd Elmoety, A.A., Hamza, Y., et al. (2017) Endoscopic Variceal Ligation Followed by Argon Plasma Coagulation against Endoscopic Variceal Ligation Alone: A Randomized Controlled Trial. Journal of Clinical Gastroenterology, 51, 49-55. https://doi.org/10.1097/MCG.0000000000000535 
Q. H. Fu

\section{Abbreviations}

AristATMAH: AristaTM Absorbable Hemostat,

AS: aethoxysklerol,

SM: sodiummorrhuate,

HP: heat probe,

APC: Argon Plasma Coagulator,

YAG: yttrium aluminum garnet,

EST: endoscopicsphincterotomy,

EIS: Endoscopic injection sclerotherapy,

STS: sodiumtetradecylsulfonate,

EVL: Endoscopic Variceal Ligation,

EUS: Endoscopic Ultrasonography. 